\title{
A missense TGFB2 variant $p .($ Arg320Cys) causes a paradoxical and striking increase in aortic TGFB1/2 expression
}

\author{
Raya Al Maskari ${ }^{1}$, Yasmin ${ }^{1}$, S Cleary ${ }^{1}$, Nikki Figg ${ }^{1}$, Sarju Mehta ${ }^{2}$, Doris Rassl ${ }^{3}$, Ian Wilkinson ${ }^{1}$ \\ and Kevin M O'Shaughnessy ${ }^{\star, 1}$
}

Loeys-Dietz syndrome (LDS) is an autosomal dominant connective tissue disorder with a range of cardiovascular, skeletal, craniofacial and cutaneous manifestations. LDS type 4 is caused by mutations in TGF $\beta$ ligand 2 (TGFB2) and based on the family pedigrees described to date, appears to have a milder clinical phenotype, often presenting with isolated aortic disease. We sought to investigate its molecular basis in a new pedigree. We identified a missense variant p.(Arg320Cys) (NM_003238.3) in a highly evolutionary conserved region of TGFB2 in a new LDS type 4 pedigree with multiple cases of aortic aneurysms and dissections. There was striking upregulation of TGFB1 and TGFB2 expression on immunofluorescent staining, and western blotting of the aortic tissue from the index case confirming the functional importance of the variant. This case highlights the striking paradox of predicted loss-of-function mutations in TGFB2 causing enhanced TGF $\beta$ signaling in this emerging familial aortopathy.

European Journal of Human Genetics (2017) 25, 157-160; doi:10.1038/ejhg.2016.143; published online 26 October 2016

\section{INTRODUCTION}

Loeys-Dietz syndrome (LDS) is an inherited autosomal dominant (AD) systemic disorder with a broad phenotypic spectrum of cardiovascular, skeletal, craniofacial and cutaneous manifestations (OMIM \#609192). The hallmark of early and progressive aortic root dilatation predisposes to premature death from dissection and rupture of the aorta. ${ }^{1}$ Other classic features include widespread arterial tortuosity, bicuspid aortic valve, bifid uvula/cleft palate and hypertelorism. ${ }^{1}$ LDS is caused by disruption to the transforming growth factor beta (TGF $\beta$ ) signaling pathway. TGF $\beta$ proteins regulate key processes including cell proliferation, angiogenesis and matrix turnover by signaling through serine/threonine kinase receptors (TGFBR1, TGFBR2) and downstream effectors, the SMAD proteins. ${ }^{2}$ TGF $\beta$ is synthesized as a dimer bound to a latencyassociated peptide (LAP) that prevents the cytokine from binding to its receptors. ${ }^{3}$ Complex mechanisms also control TGF $\beta$ sequestration and release by the extracellular matrix $(\mathrm{ECM}) .^{3}$

Mutations in TGFBR1, TGFBR2, TGF $\beta$ ligands 2 and 3 (TGFB2, TGFB3), and SMAD3 are associated with LDS disease pathogenesis. ${ }^{4-7}$ Precise genotype-phenotype correlations are still lacking, but it is proposed that a mutation in any of these genes plus arterial aneurysm/ dissection or a family history of LDS is sufficient for the diagnosis. ${ }^{8}$ LDS type 4 is caused by mutations in TGFB2 and represents a milder end of the LDS spectrum often with isolated aortic disease presenting in the mid 30s. ${ }^{9}$ To date, less than 20 mutations in TGFB2 have been identified usually in the LAP domain of the protein. ${ }^{6,9-13}$ However, the underlying pathogenic mechanisms remain unclear. Most of the mutations are predicted to be loss-of-function, but their downstream effect appears to be a paradoxical activation of TGF $\beta$ signaling. ${ }^{14}$

Here we report a new pedigree with LDS4 and confirm that the causative variant p.(Arg320Cys) (NM_003238.3) causes striking upregulation of TGFB1/2 expression in the aorta. This confirms that the variant is functional and corroborates previous reports of an enhanced aortic TGF $\beta$ 'tissue signature' in LDS and other TGF $\beta$ vasculopathies.

\section{MATERIALS AND METHODS}

Data submission

The phenotype and variant data were submitted into LOVD v.3.0 Build 16 http://medgen.ua.ac.be/LOVDv.3.0/individuals/00000322.

\section{Control subjects}

Formalin-fixed and paraffin-embedded (FFPE) aortic tissue from ageand gender-matched donors $(n=5)$ were obtained from the Transplant service at Addenbrooke's Hospital (Cambridge, UK). All samples were handled in accordance with the policies and procedures of the Human Tissue Act and had Local and Regional Ethics approval.

\section{Immunofluorescence staining}

The FFPE sections of the surgical specimen were deparaffinised in Histoclear (National Diagnostics, Atlanta, GA, USA) then dehydrated through graduated methanols. Antigen retrieval was performed in $\mathrm{pH}$ 6 citrate buffer (Vector Laboratories Ltd, Peterborough, UK) using 2100 Retriever (Aptum Biologics Ltd, Southampton, UK). Sections were permeabilised with $0.05 \% \mathrm{v} / \mathrm{v}$ Triton X-100-PBS for $5 \mathrm{~min}$ and blocked for $2 \mathrm{~h}$ at room temperature with $5 \% \mathrm{v} / \mathrm{v}$ goat serum in $0.05 \%$

\footnotetext{
${ }^{1}$ Department of Medicine (EMIT and CVD divisions), University of Cambridge, Cambridge, UK; ${ }^{2}$ Department of Medical Genetics, Addenbrookes Hospital, Cambridge, UK; ${ }^{3}$ Department of Pathology, Papworth Hospital, Cambridge, UK

${ }^{*}$ Correspondence: Dr K O'Shaughnessy, Department of Medicine (EMIT Div) University of Cambridge Clinical School Addenbrookes Hospital, Box 110, Level 6 ACCI, Cambridge CB2 2QQ, UK. Tel: +441223762578; Fax: +441223762576; E-mail: kmo22@medschl.cam.ac.uk

Received 26 April 2016; revised 19 August 2016; accepted 20 September 2016; published online 26 October 2016
} 
a

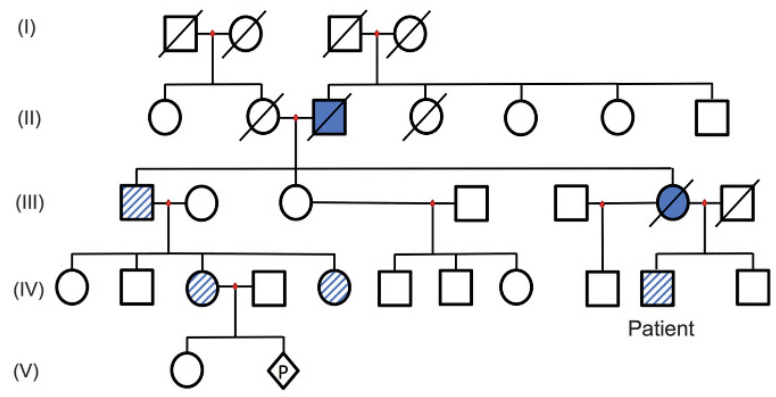

$\square$ Male $\bigcirc$ Female $\diamond$ Pregnant $\square$

Aneurysm/Dissection $\oslash$ Variant positive b

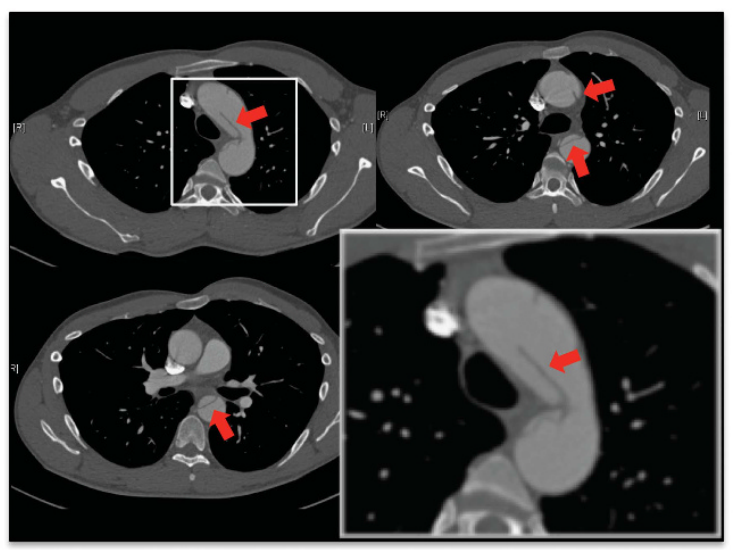

d
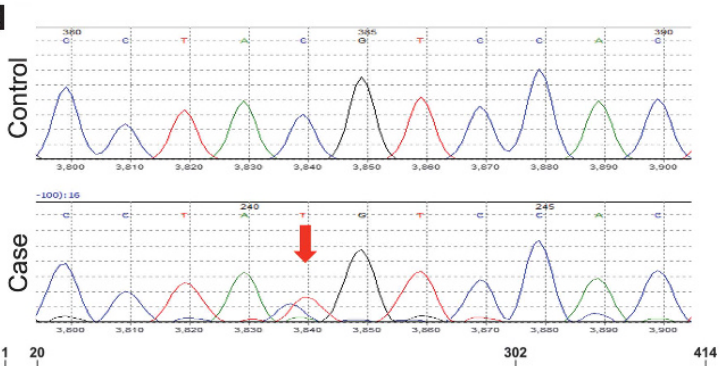

SP

SP
TGFb2 cytokine

Figure 1 Clinical and Molecular Findings. (a) Family pedigree with multiple cases of aneurysms and dissections suggesting autosomal dominant inheritance. The variant was detected in the index case (IV:10) and III:1, IV:3 and IV:5. (b) CT images showing an intimal flap (red arrows) extending from the aortic valve into the thoracic aorta. (c) Elastin staining of IV:10 aorta showing extensive elastic fibre fragmentation at the site of dissection (scale bar $=6 \mathrm{~mm}$ ). (d) Sequencing chromatogram showing a missense variant (c.958C > T) in exon 6 of TGFB2. The variant is in a region of TGFB2 that is highly conserved across species. LAP, latency-associated peptide; SP, signal peptide.

$\mathrm{v} / \mathrm{v}$ Triton X-100-PBS. Sections were probed with mouse monoclonal to TGFB2 (Abcam, Cambridge, UK) and mouse monoclonal to TGFB1 (Abcam) for $16 \mathrm{~h}$ at $4{ }^{\circ} \mathrm{C}$ at $1: 200$ dilution in $2 \% \mathrm{v} / \mathrm{v}$ goat serum in $0.05 \% \mathrm{v} / \mathrm{v}$ Triton X-100-PBS. Slides were then washed for $5 \mathrm{~min}$ in $0.05 \% \mathrm{v} / \mathrm{v}$ Triton X-100-PBS and incubated in secondary antibody for $1 \mathrm{~h}$ at room temperature. Pre-absorbed goat IgG-conjugated Alexa Fluor 633 secondary antibody (ThermoFisher Scientific, Waltham, MA, USA) was used at 1:200 dilution in $2 \% \mathrm{v} / \mathrm{v}$ goat serum in $0.05 \% \mathrm{v} / \mathrm{v}$ Triton $\mathrm{X}-100-\mathrm{PBS}$. Sections were counterstained with Sytox Orange (ThermoFisher Scientific) at 1/10 000 in Milli-Q water for $20 \mathrm{~min}$ at room temperature then mounted with ProLong Gold Antifade Mountant (ThermoFisher Scientific).

Images were acquired with a Leica SP8 (Leica Microsystems, Wetzlar, Germany) inverted laser scanning confocal microscope using a $20 \times 1.4$ N.A. dry objective. Acquisition parameters were: 12-bit, $1024 \times 1024$ pixels, $1.25 \times$ and $3 \times$ digital zooms, $8000 \mathrm{~Hz}$ scan speed, 16-line Kalman filtering and 2 frame accumulation. All images were acquired using identical scan settings.
Protein extraction and western blotting

Western blotting for TGFB1 and TGFB2 was performed in the case and two of the controls. Three $15 \mu \mathrm{m}$ FFPE sections of each tissue sample were deparaffinised in Histoclear 3 times. The procedure was serially repeated with 100, 95 and 70\% ethanol, washing twice for 10 min each. Pellets were air dried, resuspended in Extraction Buffer EXB Plus (Qiagen, Hilden, Germany) containing $\beta$-mercaptoethanol and incubated at $4{ }^{\circ} \mathrm{C}$ for $5 \mathrm{~min}$, then at $100^{\circ} \mathrm{C}$ for $20 \mathrm{~min}$ followed by a $2 \mathrm{~h}$ incubation at $80^{\circ} \mathrm{C}$ with agitation and a final incubation at $4{ }^{\circ} \mathrm{C}$ for $1 \mathrm{~min}$. Samples were then centrifuged at $4{ }^{\circ} \mathrm{C}$ for $15 \mathrm{~min}$ and the protein was quantified using Pierce BCA protein assay (ThermoFisher Scientific) and stored at $-70^{\circ} \mathrm{C}$ until further use.

For the western blot, $10 \mu \mathrm{g}$ of protein lysates were used. Samples were incubated at $70{ }^{\circ} \mathrm{C}$ for $10 \mathrm{~min}$ in Lithium dodecylsulfate (LDS) sample loading buffer (ThermoFisher Scientific) and Bolt sample reducing agent (ThermoFisher Scientific). Samples for TGFB1 blotting were performed under non-reducing conditions. Protein was separated by SDS-gel electrophoresis in $4-12 \%$ gradient Bis-Tris Plus Bolt gels (ThermoFisher Scientific) at $200 \mathrm{~V}$ for $30 \mathrm{~min}$ and transfered to 
$0.22 \mu \mathrm{M}$ nitrocellulose membrane (ThermoFisher Scientific) using the iBlot2 dry blotting system (ThermoFisher Scientific) at $20 \mathrm{~V}$ for $10 \mathrm{~min}$. Prior to transfer, gels were equilibrated for $5 \mathrm{~min}$ in NuPage transfer buffer (ThermoFisher Scientific) containing 10\% methanol. Membranes were blocked with 5\% w/v milk in TBS buffer for $1 \mathrm{~h}$ at room temperature then incubated with primary antibodies in $5 \%$ milk $\mathrm{w} / \mathrm{v}$ in $0.1 \% \mathrm{v} / \mathrm{v}$ Tween 20 -TBS for $16 \mathrm{~h}$ at $4{ }^{\circ} \mathrm{C}$. Anti-TGFB2 (Abcam) rabbit polyclonal IgG antibody was used at 1:500 dilution and $\beta$-actin mouse monoclonal IgG (ThermoFisher Scientific) was used as a loading control at 1:1000 dilution. Anti-TGFB1 (Abcam) mouse monoclonal IgG antibody was used at 1:500 dilution and $\beta$-actin rabbit polyclonal IgG (Sigma Aldrich, St Louis, MO, USA) was used as a loading control at 1:1000 dilution. Secondary antibodies were incubated in $0.1 \% \mathrm{v} / \mathrm{v}$ Tween 20 -TBS for $1 \mathrm{~h}$ at room temperature. Donkey anti-rabbit (LI-COR Biotechnology UK Ltd, Cambridge, UK) IRDye $800 \mathrm{CW}$ and goat anti-mouse (ThermoFisher Scientific) Alexa Fluor 680-conjugated secondary antibodies were used at 1:5000 dilution. Membranes were washed in $0.1 \% \mathrm{v} / \mathrm{v}$ Tween 20-TBS 3 times for 15 min each between primary and secondary antibody incubations, and before visualization. Protein bands were detected using the LICOR Odessey system. Signal intensities were normalised against $\beta$ actin and quantified using ImageStudioLite software.

\section{RESULTS}

\section{Pedigree discovery}

A 27-year-old man presented with severe pain radiating down his back after lifting a lawn mower into a van. The family history revealed several family members with aortic aneurysms and dissections: his mother (III:6) died following an aortic dissection, a maternal uncle (III:1) had emergency repair of an aortic aneurysm and his maternal grandfather (II:3) had a dissection of an abdominal aortic aneurysm and separate iliac artery aneurysms (Figure 1a). On examination, the only sign was an elevated BP of $240 / 100 \mathrm{~mm} \mathrm{Hg}$. A CT scan with contrast showed a Stanford type-A dissection with an intimal flap extending the full length of the aorta from the aortic valve into both iliac arteries (Figure 1b). He underwent open aortic repair and a surgical specimen was recovered for further examination. Histologic examination showed fragmentation and disruption of the aortic elastic fibers and cystic medial necrosis (Figure 1c).

\section{Sequencing and variant identification}

DNA sequencing from the peripheral blood of the index case identified a missense variant (c.958C $>$ T) (NM_003238.3) in exon 6 of the TGFB2 gene (NG_027721.1). This variant causes a p. (Arg320Cys) substitution in a highly conserved region of TGFB2 (Figure 1d). The variant was also detected in the uncle (III:1) and two currently asymptomatic teenage family members (IV:3) and (IV:5).

\section{TGFB1 and TGFB2 expression in the aorta}

Immunofluorescent imaging showed markedly enhanced TGFB1 and TGFB2 expression in the aorta of the index case compared with age-and gender-matched controls (Figures 2 and 3). This was confirmed by immunoblotting for both proteins in the aorta of IV:10 versus controls where the upregulation of TGFB1 was particularly striking (Figures 2 and 3).

\section{DISCUSSION}

The p.(Arg320Cys) substitution is in a highly evolutionarily conserved region of TGFB2 and has a strong in silico prediction for pathogenicity. ${ }^{11}$ However, there has been no evidence to confirm its functional effects. ${ }^{11}$ We show for the first time that this variant does induce striking upregulation of both TGFB1 and TGFB2 in the vessel wall of a subject with the variant. While this is unexplained by a lossof-function variant in TGFB2, this signature of enhanced TGF $\beta$ signaling is believed to have a central role in the aortic dilatation and aneurysms seen in LDS, Marfan syndrome and other inherited aortopathies. ${ }^{15}$

Of note, haploinsufficient TGFB2 ${ }^{+/-}$mice develop aortic root dilatation and aneurysm, and have a higher expression of phosphorylated SMAD2/3 and extracellular signal-regulated kinases (ERK1/2), indicating upregulation of the TGF $\beta$ canonical (SMAD dependent) and non-canonical (SMAD independent) pathways. ${ }^{6}$ The canonical pathway is involved in stimulating elastin and collagen while repressing ECM degradation by inducing endogenous tissue inhibitor of metalloproteinases 1 and 3 . These changes disrupt the normal a
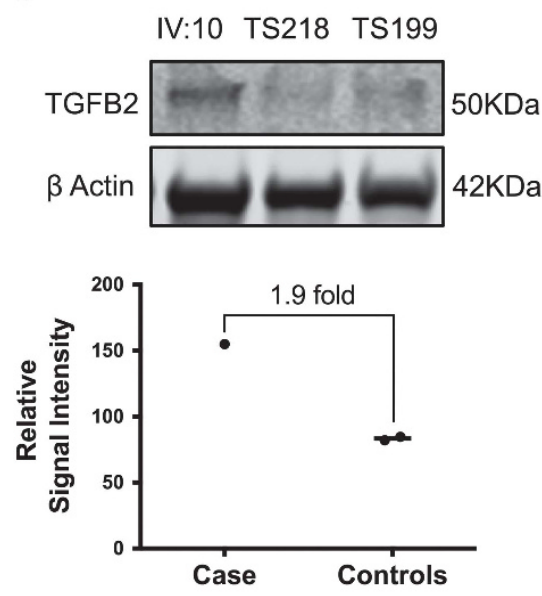
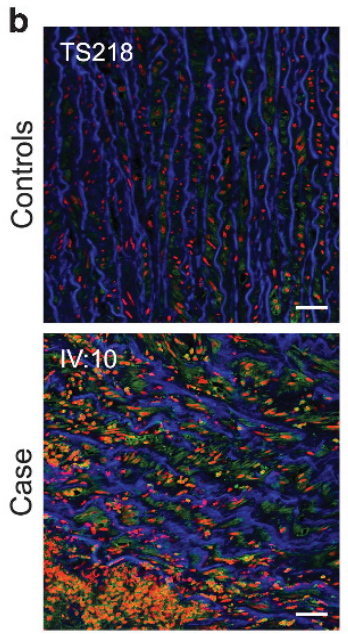
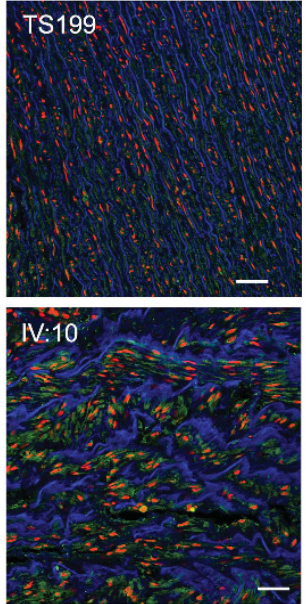

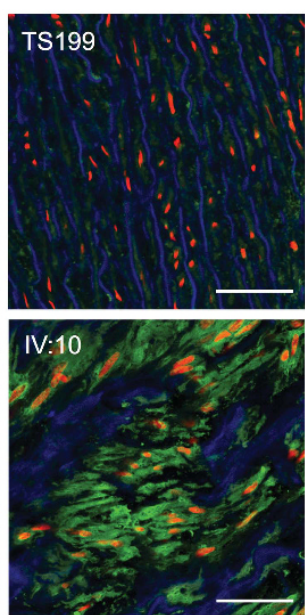

Figure 2 Aortic Expression of TGFB2. (a) Western blots of aortic TGFB2 expression in IV:10 compared with two age-matched controls, TS218 and TS199. Signal intensities of TGFB2 normalized against $\beta$-actin indicate a two-fold higher expression of TGFB2 in the case. (b) Immunofluorescent staining of TGFB2 (green) shows increased expression in the aortic wall of IV:10 compared with TS218 and TS199. Elastic fibres appear as blue (autofluorescence) and nuclei are counterstained red (scale bar $=50 \mathrm{uM}$ and the right-hand images are zoomed). 
a

\section{IV:10 TS218 TS199}
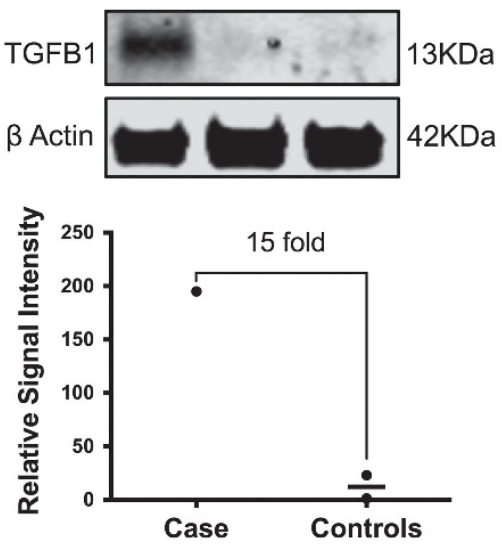
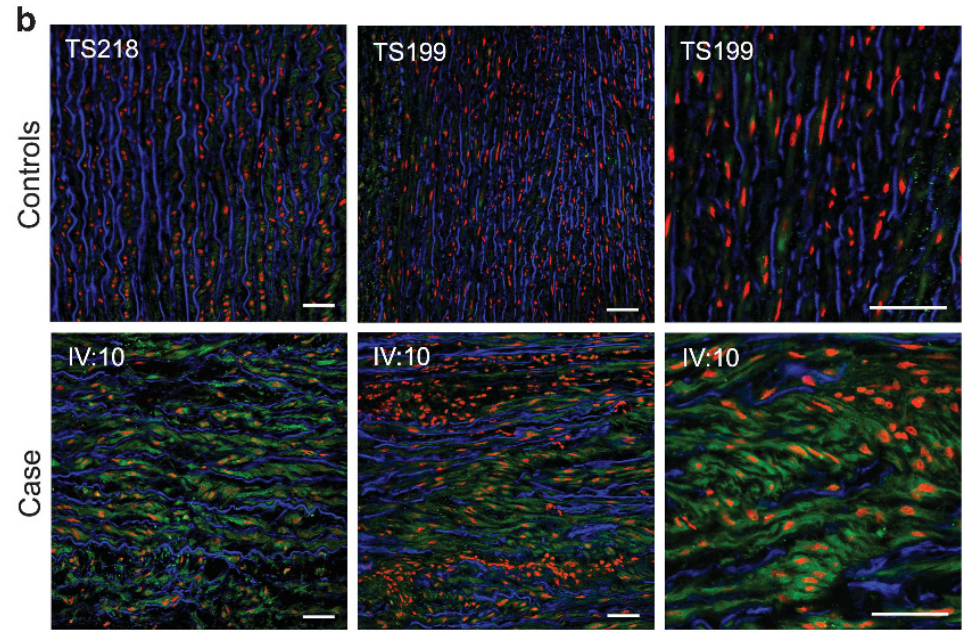

Figure 3 Aortic Expression of TGFB1. (a) Western blots of aortic TGFB1 expression in IV:10 compared with two age-matched controls, TS218 and TS199. Signal intensities of TGFB1 normalized against $\beta$-actin indicate a 15-fold higher expression of TGFB1 in the case. (b) Immunofluorescent staining of TGFB1 (green) shows increased expression in the aortic wall of IV:10 compared with TS218 and TS199. Elastic fibres appear as blue (autofluorescence) and nuclei are counterstained red (scale $\mathrm{bar}=50 \mathrm{uM}$ and the right-hand images are zoomed).

architecture of the vessel wall. ${ }^{2}$ Less is known about the role of the non-canonical pathway: however, enhanced ERK activity appears to stimulate the expression of matrix metalloproteinases 2 and 9 to stimulate matrix degradation. ${ }^{2,15}$

Another suggestion to resolve the TGF $\beta$ vascular paradox, posits that other TGFB ligands are overexpressed to compensate for haploinsufficiency of a given TGFB ligand. This shift in ligand usage is seen with higher TGFB1 expression in TGFB2 ${ }^{+/-}$mice $^{6}$ and in our index case. The high TGFB1/2 expression may even be a 'repair process' by mesenchymal cells following damage to ECM. ${ }^{3}$

As aortic aneurysm and dissection may be the only manifestation of LDS4, identifying family members with the variant is crucial for surveillance to improve clinical outcome. The striking activation of $\operatorname{TGF} \beta$ signaling in these patients also suggests this may be a future therapeutic target for LDS.

\section{CONFLICT OF INTEREST}

The authors declare no conflict of interest.

\section{ACKNOWLEDGEMENTS}

Raya $\mathrm{Al}$ Maskari has a $\mathrm{PhD}$ studentship funded by the Omani government.

\section{AUTHOR CONTRIBUTIONS}

RAM, Y, SC and NF did the bench work; RAM did all the confocal imaging and blotting; SM provided medical genetics diagnostics and advice; DZ provided pathology services and advice; IB identified the pedigree and gave clinical advice; KMO conceived and ran the project and wrote the manuscript with RAM.
1 Loeys BL, Dietz HC: Loeys-Dietz Syndrome. In: Pagon RA AM, Ardinger HH (eds): GeneReviews (Internet). University of Washington, Seattle: Seattle, WA, USA, 2013.

2 Jones JA, Spinale FG, Ikonomidis JS: Transforming growth factor-beta signaling in thoracic aortic aneurysm development: a paradox in pathogenesis. J Vasc Res 2009; 46: 119-137.

3 Horiguchi M, Ota M, Rifkin DB: Matrix control of transforming growth factor-beta function. J Biochem 2012; 152: 321-329.

4 Loeys BL, Chen J, Neptune ER et al: A syndrome of altered cardiovascular, craniofacial, neurocognitive and skeletal development caused by mutations in TGFBR1 or TGFBR2. Nat Genet 2005; 37: 275-281.

5 van de Laar IM, Oldenburg RA, Pals G et al: Mutations in SMAD3 cause a syndromic form of aortic aneurysms and dissections with early-onset osteoarthritis. Nat Genet 2011; 43: 121-126.

6 Lindsay ME, Schepers D, Bolar NA et al: Loss-of-function mutations in TGFB2 cause a syndromic presentation of thoracic aortic aneurysm. Nat Genet 2012; 44: 922-927.

7 Bertoli-Avella AM, Gillis E, Morisaki $\mathrm{H}$ et al: Mutations in a TGF-beta ligand, TGFB3, cause syndromic aortic aneurysms and dissections. J Am Coll Cardiol 2015; 65: 1324-1336

8 MacCarrick G, Black 3rd JH, Bowdin S et al: Loeys-Dietz syndrome: a primer for diagnosis and management. Genet Med 2014; 16: 576-587.

9 Boileau C, Guo DC, Hanna N et al: TGFB2 mutations cause familial thoracic aortic aneurysms and dissections associated with mild systemic features of Marfan syndrome. Nat Genet 2012; 44: 916-921.

10 Ritelli M, Chiarelli N, Dordoni C et al: Further delineation of Loeys-Dietz syndrome type 4 in a family with mild vascular involvement and a TGFB2 splicing mutation. BMC Med Genet 2014; 15: 91.

11 Gago-Diaz M, Blanco-Verea A, Teixido-Tura G et al: Whole exome sequencing for the identification of a new mutation in TGFB2 involved in a familial case of non-syndromic aortic disease. Clin Chim Acta 2014; 437: 88-92.

12 Leutermann R, Sheikhzadeh S, Brockstadt L et al: A 1- bp duplication in TGFB2 in three family members with a syndromic form of thoracic aortic aneurysm. Eur J Hum Genet 2014; 22: 944-948.

13 Renard M, Callewaert B, Malfait F et al: Thoracic aortic-aneurysm and dissection in association with significant mitral valve disease caused by mutations in TGFB2. Intern J Cardiol 2013; 165: 584-587.

14 Akhurst RJ: The paradoxical TGF-beta vasculopathies. Nat Genet 2012; 44: 838-839.

15 Lindsay ME, Dietz HC: Lessons on the pathogenesis of aneurysm from heritable conditions. Nature 2011; 473: 308-316. 